Research Paper

\title{
Functional Role of HSP47 in the Periodontal Ligament Subjected to Occlusal Overload in Mice
}

\author{
Hiroaki Mimura ${ }^{1}$, Tatsuo Takaya ${ }^{1}$, Saeka Matsuda², Keisuke Nakano ${ }^{3}$, Rina Muraoka ${ }^{4}$, Mihoko Tomida , \\ Norimasa Okafuji², Takeo Fujii ${ }^{1}$, and Toshiyuki Kawakami2 ${ }^{\circledR}$ \\ 1. Department of Oral Health Promotion, Matsumoto Dental University Graduate School of Oral Medicine, Shiojiri, Japan \\ 2. Department of Hard Tissue Research, Matsumoto Dental University Graduate School of Oral Medicine, Shiojiri, Japan \\ 3. Department of Oral Pathology, Okayama University Graduate School of Medicine, Dentistry and Pharmaceutical Sciences, Okayama, Japan \\ 4. Department of Orthodontics, Matsumoto Dental University School of Dentistry, Shiojiri, Japan \\ 5. Department of Oral and Maxillofacial Biology, Matsumoto Dental University Graduate School of Oral Medicine, Shiojiri, Japan \\ $\bowtie$ Corresponding author: kawakami@po.mdu.ac.jp
}

() Ivyspring International Publisher. Reproduction is permitted for personal, noncommercial use, provided that the article is in whole, unmodified, and properly cited. See http://ivyspring.com/terms for terms and conditions.

Received: 2015.10.15; Accepted: 2016.01.22; Published: 2016.02.20

\begin{abstract}
We carried out an experiment to induce traumatic occlusion in mice periodontal tissue and analyzed the expression of HSP47. Continuous traumatic occlusion resulted to damage and remodeling of periodontal ligament as well as increase in osteoclasts and bone resorption. Four days after traumatic occlusion, osteoclasts did not increase but Howship's lacunae became enlarged. That is, the persistent occlusal overload can destroy collagen fibers in the periodontal ligament. This was evident by the increased in HSP47 expression with the occlusal overload. HSP47 is maintained in fibroblasts for repair of damaged collagen fibers. On the other hand, osteoclasts continue to increase although the load was released. The osteoclasts that appeared on the alveolar bone surface were likely due to sustained activity. The increase in osteoclasts was estimated to occur after load application at day 4. HSP47 continued to increase until day 6 in experiment 2 but then reduced at day 10. Therefore, HSP47 appears after a period of certain activities to repair damaged collagen fibers, and the activity was returned to a state of equilibrium at day 30 with significantly diminished expression. Thus, the results suggest that HSP47 is actively involved in homeostasis of periodontal tissue subjected to occlusal overload.
\end{abstract}

Key words: Occlusal trauma, Periodontal ligament, Immunohistochemistry, HSP47, Mouse

\section{Introduction}

Regarding the examination results of periodontal ligament in experimental occlusal trauma mouse model, we have reported the cytological behavior of the related regions. Periodontal connective tissue remodeling was occurred due to traumatic occlusal overload (1). In the remodeling course, the fibroblasts act as an important roles: such as collagen synthesis. Heat shock protein 47 (HSP47) is a collagen-binding stress protein that acts as a collagen-specific molecular chaperone during the biosynthesis and secretion of procollagen. Type I collagen is a major component of periodontal ligaments (2-4). Thus, in the examination, we have examined the immunohistochemical expression of HSP47 in the experimentally induced traumatic occlusal periodontal ligament tissue.
We developed an experimental model by exposing the periodontal tissue to occlusal overload and the expression of heat shock protein (HSP) was determined using immunohistochemistry (IHC). The results showed a marked increase in fibroblasts in response to excessive occlusal load. HSP47 is considered to play an important role in the maintenance of homeostasis in fibroblasts exposed to traumatic occlusion. Expressions of HSP27 and HSP70 have been observed by Muraoka et al. (5-7) during injury to the periodontal tissue. We followed the experiment of Fujii et al. (8) and Takaya et al. (1) and performed a histological and IHC study on mouse periodontal tissue. 


\section{Materials and Methods}

A total of $30 \mathrm{ddY}$ mice (Japan SLC Inc, Hamamatsu), age 7-week old, with a weight of $35 \pm 2$ g were used in the experiment. The animals were housed in an air- conditioned room maintained at $24 \pm 1{ }^{\circ} \mathrm{C}$ and placed in plastic cages lined with bedding (Paper clean: Paperlet Co., Ltd. Hamamatsu). The animals had free access to solid feed (Picolab Rodent Diet 20: Nippon SLC Co., Ltd, Hamamatsu) and water. Moreover, there was no major change in the weight of the animals during the experimental period.

The experiment was carried out by placing the animals under general anesthesia using $40 \mathrm{mg} / \mathrm{kg}$ of Somnopentyl (Pentobarbital Sodium, Kyoristu Seiyaku Co., Tokyo) and then fixed on a homemade bench in a supine position. In order to keep the mouth open, the maxillary incisors were tied with a rubber band upward to the bench and a kite string was used on the lower incisors to fix the mandible. Using1/4 round bur, a hole was created on the occlusal surface of the maxillary first molar. Then after, a stainless steel micro plus screw (Osato Ltd., Saitama) with a head diameter of $1.7 \mathrm{~mm}$, head thickness of $0.5 \mathrm{~mm}$, nominal diameter of $1.0 \mathrm{~mm}$ and total length of 3.5 $\mathrm{mm}$ was implanted. This created a premature contact on the opposing mandibular first molar causing occlusal trauma. The premature contact was confirmed using micro-CT (literature added) in vivo. The experimental period lasted for 30 days and the untreated group served as the control group. Changes in the periodontal ligament were observed at 1, 4, 7 and 14. Those groups constitute experiment 1 . Then after, the micro plus screw was removed at day 4 after implantation and the subsequent tissue changes were observed (experiment 2). Therefore, the time schedule of the experiment 1 and 2 is mentioned in Table 1 . The Animal Ethics Committee of Matsumoto Dental University approved the experiment (Number \#233-13).

Table 1. Experimental Periods and Animal Number

\begin{tabular}{|c|c|c|c|c|c|c|}
\hline \multirow[t]{3}{*}{ Experiment 1} & \multicolumn{5}{|c|}{$\begin{array}{l}\text { Micro screw pins were continuously implanted for } \\
\text { each day. }\end{array}$} & \multirow[t]{2}{*}{ Total } \\
\hline & Cont & 1 day & 4 days & 7 days & 14 days & \\
\hline & 3 & 3 & 3 & 3 & 3 & 15 \\
\hline \multirow[t]{3}{*}{ Experiment 2} & \multicolumn{5}{|c|}{$\begin{array}{l}\text { Examination after micro screw pins removed at day } \\
4 .\end{array}$} & Total \\
\hline & Cont & 3 days & 6 days & 10 day & 30 days & \\
\hline & 3 & 3 & 3 & 3 & 3 & 15 \\
\hline
\end{tabular}

\section{Histopathology}

After the mandibular bone and periodontal tissue were removed, specimens were immediately fixed in $4 \%$ paraformaldehyde and $0.05 \mathrm{M}$ phosphate buffer for 10 days. Then after, specimens were decalcified in
$10 \%$ EDTA for 3 weeks. This was followed by embedding in paraffin, deparaffinization in xylene and vertical sectioning of the root portion with a thickness of $4 \mu \mathrm{m}$. Specimens were stained with hematoxylin and eosin and examined under the light microscope.

\section{Immunohistochemistry}

After deparaffinization, the slides were treated in incubator at $60^{\circ} \mathrm{C}$ for $30 \mathrm{~min}$. Specimens were subjected to proteolytic enzyme (Protease, Nichirei), immersed in $0.03 \%$ hydrogen peroxide methanol solution for $3 \mathrm{~min}$, followed by endogenous peroxidase activation for $10 \mathrm{~min}$. Anti-HSP47 (ab77609, Abcam) diluted at 2000x was the primary antibody. Polyclonal anti-rabbit (Simple Stain, Mouse MAX-PO ${ }^{\circledR}$, Tokyo, Nichirei) was the secondary antibody. Then after, slides were washed with PBS and then subjected to DAB color development for 3 min. Finally, counterstaining was done by immersing the specimen in hematoxylin for $1 \mathrm{sec}$.

\section{Results}

\section{Histopathological examination}

\section{Experiment 1}

Dense amount of periodontal ligament fibroblasts and spindle cells in the control group were seen. Capillaries were congested with red blood cells. Periodontal ligament fibers were irregularly arranged (Fig. 1A). Osteoclasts were noticeable in alveolar bone. The furcation is lined by acellular cementum.

At day 1 of experimental group, dilated capillaries were filled with red blood cells. At day 4, stronger hyperemia was observed compared to day 1 . The amount of deeply stained cells with round nuclei increased (Fig. 1B). More osteoclasts were observed on the glassy surface of the alveolar bone. At day 7, the cell density was reduced compared to day 4 . Moreover, osteoclasts appeared in between fibroblasts (Fig. 1C). Howship's lacunae formed in borders of resorbed bone and cementum (Fig. 1D). Resorbed cementum was part of the acellular cementum. At day 14 , bone resorption and osteoclasts in lacunae have become more evident compared to day 7. Resorption of cementum also increased.

\section{Experiment 2}

Strong hyperemia was seen at day 4 in the control group (experimental group 1). Significant vasodilation was observed in the experimental group at day 3 compared to control group. Osteoclasts were scattered in the alveolar bone and some formed clusters in Howship's lacunae (Fig. 2A). At day 6, capillaries were less indicating a decrease in hyperemia and fewer osteoclasts were noted compared to day 3 . 
Fibroblasts were deeply stained with hematoxylin stain and have round nucleus. At day 10, more Howship's lacunae were observed compared to day 6. At day 30, fibroblasts and dilated capillaries were no longer conspicuous. The histological findings are similar to the non-treated or control group in experiment 1 (Fig. 2B).

\section{Immunohistochemistry}

\section{Experiment 1}

HSP47 was slight detected in the cytoplasm of fibroblasts in the control group (Fig. 3A). Other cells that were scattered in the periodontal ligament were also positive to HSP47. The protein was mainly de- tected in the cytoplasm (Fig. 3B). At day 1, HSP47 expression was detected more in fibroblasts in epithelial attachment, the intensity was similar to those in the control group. At day 4, HSP47 was detected in the entire periodontal ligament and the intensity was stronger compared to the control group. In particular, intense expression was detected in fibroblasts lining the alveolar bone (Fig. 3C). HSP47 expression further increased at day 7 compared to day 4 . HSP47 was also detected in vascular endothelial cells. The strongest expression was detected in cells at day 14 (Fig. 3D). Furthermore, the percentage of cells that expressed the protein also increased.

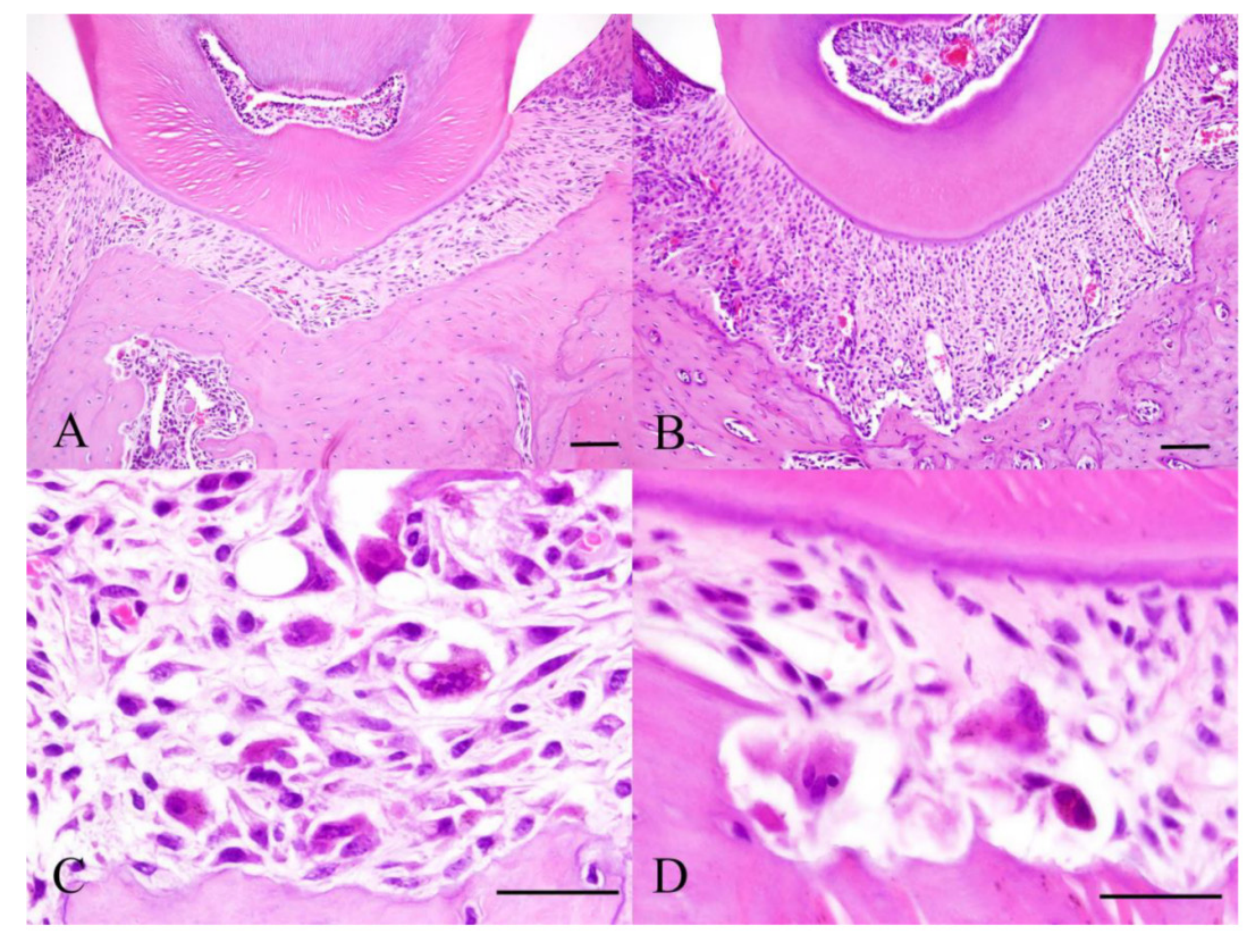

Figure 1. Histopathology of Experiment 1. Control specimen (A), Experimental day 4 specimen (B), Experimental day 7 specimen (C) and Experimental day 14 specimen (D). Scale bar: $50 \mu \mathrm{m}$.

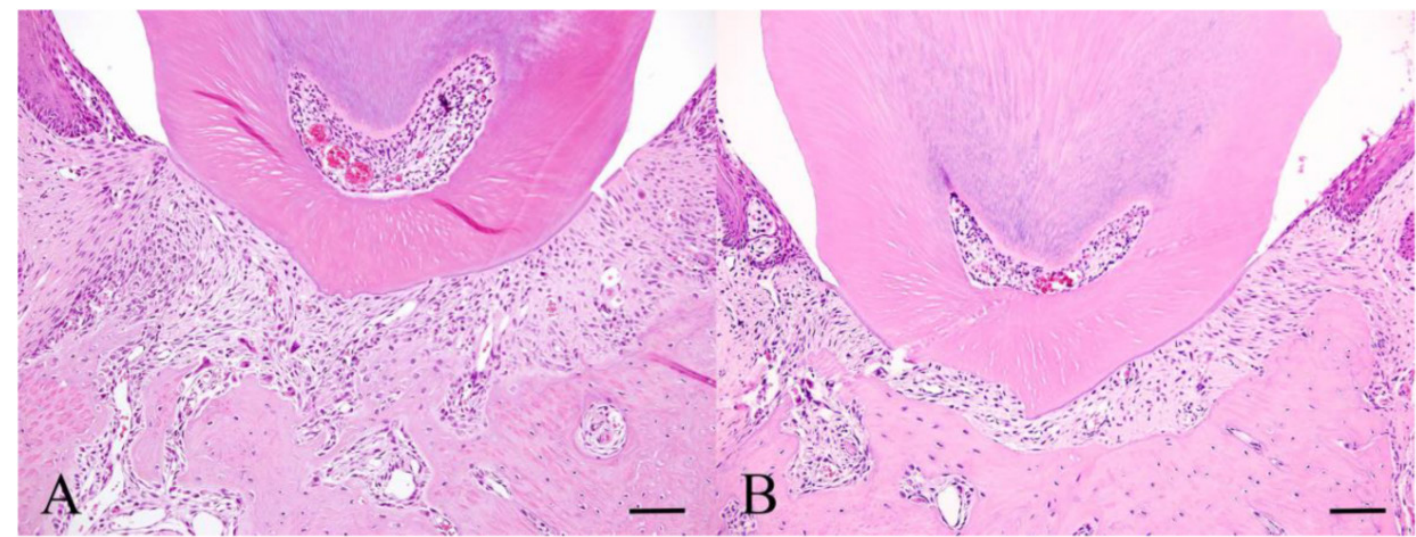

Figure 2. Histopathology of Experiment 2. Experimental day 3 specimen (A) and Experimental day 30 specimen (B). Scale bar: $50 \mu \mathrm{m}$. 


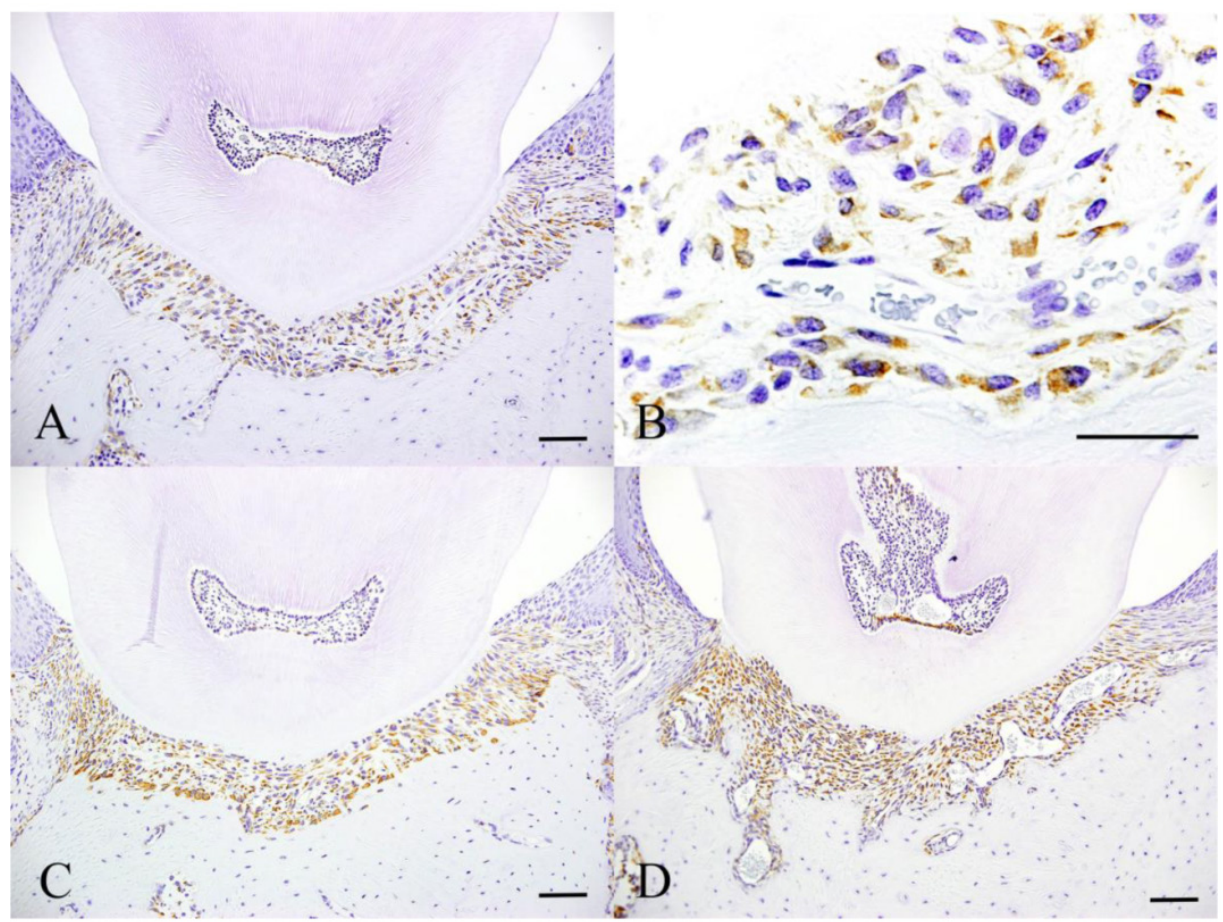

Figure 3. Immunohistochemical features of Experiment 1. Control specimen (A and B), Experimental day 4 specimen (C) and Experimental day 14 specimen (D). Scale bar: $50 \mu \mathrm{m}$.

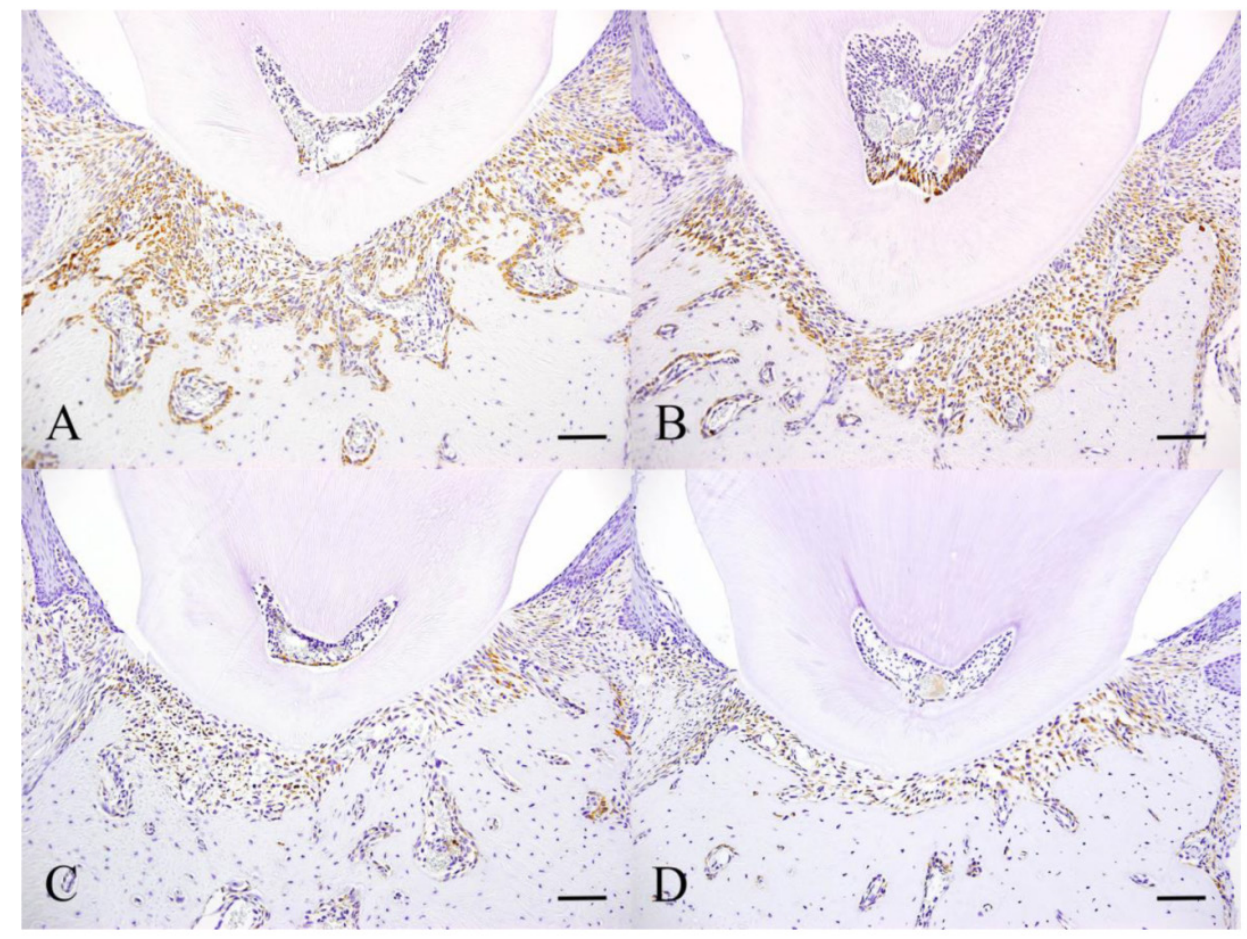

Figure 4. Immunohistochemical features of Experiment 2. Experimental day 3 specimen (A), Experimental day 6 specimen (B), Experimental day 10 specimen (C) and Experimental day 30 specimen. Scale bar: $50 \mu \mathrm{m}$.

\section{Experiment 2}

The control group is the same as day 4 in experiment 1 . At day 3, the number of fibroblasts that expressed HSP47 was similar to the control group (Fig. 4A). Some cells scattered in the periodontal ligament also expressed HSP47. The cells that expressed the protein in the control group are neatly arranged on the alveolar bone surface. The intensity of expression at day 6 increased compared to day 3 (Fig. $4 \mathrm{~B})$. The majority of cells that were positive have round nuclei. At day 10, the number of cells showing 
positive reaction decreased compared to day 6 (Fig. $4 \mathrm{C}$ ). At day 30 , only few scattered cells were positive and this was comparable to the control group in experiment 1 (Fig. 4D).

\section{Discussion}

It has been known that excessive occlusal force and occlusal trauma has a long- term effect on the periodontal ligament. Moreover, occlusal trauma has destructive effect on periodontal tissue (9-12). Various studies have been conducted on the relationship between traumatic occlusion and bone resorption. According to Glickman et al. (13), in severe periodontitis with advanced bone resorption, inflammatory changes are caused by pathogenic bacteria but are highly influenced by both occlusal trauma and traumatic occlusion. However, according to Wearhaug et al. (14), occlusal trauma does not participate in connective tissue damage and bone resorption. Subsequent animal studies have been conducted to determine the relationship between traumatic occlusion and periodontitis (15-20). Although recent studies mentioned the anatomical pathology of the tissue, the severity of periodontitis with advanced bone resorption caused by occlusal trauma has not been definitively confirmed.

An immunohistochemical study on periodontal tissue was performed using new experimental system in vivo (1). This experiment by Kaku et al. (21) was carried out in rats in which excessive occlusal load was applied. In the present experiment, a micro plus screw with a standard head diameter was implanted in the maxillary first molar to produce a uniformly high occlusal contact. Furthermore, it was possible to reduce the torque by tightening the micro plus screw during the experimental period. Since the gliding movement of the lower jaw in mouse is relatively simple, creating a premature contact to produce excessive load on the molar was also easy. Results showed that remodeling of periodontal ligament from day 4 was rapidly reduced due to occlusal trauma. The increase in fibroblasts as part of the remodeling of periodontal tissue was suggested to be due to adaptation to excessive load (1). In this regard, we considered that the histological changes in fibroblasts would significantly increase at day 4 , and so we prepared another experimental system. In other words, implantation of the micro plus screw to produce traumatic occlusion was only allowed at day 4 and then, histological and expression of HSP47 were examined after traumatic occlusion.

The periodontal ligament at the furcation area in the control group in experiment 1 runs in an orderly fashion from the tooth to the alveolar bone but the fibroblasts were sort of irregular. It can be speculated that the occlusal force to the furcation was in an equilibrium state. In experiment 1, it was inferred that congestion and vasodilation occurred from day 1 . Since there was an increase in hyperemia at day 4 , excessive occlusal load caused tissue reaction by a rise in blood supply. However, no change in the capillaries was seen at day 7 . This is considered to be affected by HSP47 which will be discussed later. On the other hand, osteoclasts in Howship's lacunae as well as bone resorption continued to rise. Takaya et al mentioned that the osteoclasts which appeared after occlusal trauma were derived from bone marrow. Considering this, the osteoclasts observed at day 7 were probably bone marrow derived cells. With the enlargement of Howship's lacunae at day 14 compared to day 7 , this suggests that the activity of the osteoclasts was due to excessive load.

At day 3 in experiment 2, hyperemia was not so prominent compared to control group and when occlusal overload was released, hyperemia declined as well. At day 6 and 10, osteoclasts were reduced compared to day 3. Therefore, the osteoclasts were induced by the implantation of micro plus screw at day 4 , and their activity continued even if the micro plus screw was removed. Meanwhile, the constant activity of osteoclasts was brought about by the influence of occlusal overload which allowed the cells to proceed to the activity which already began. This was shown by the enlargement of Howship's lacunae at day 10 compared to day 6 . This means that the increase in osteoclasts was due to occlusal overload in experiment 1 and continued to increase even when the load was removed in experiment 2 . At day 30 , congestion nor vasodilation was no longer observed having similar histological features to control group. It can be inferred that at day 30, the periodontal ligament was restored to its equilibrium state.

HSP47 or heat shock protein is a major protein that is expressed in various tissues and organs. This is seen in pathological changes such as ischemia, infection, inflammation, radiation, physical stress to light, enzyme stress such as heavy metal ions, arsenic, arsenite, ethanol and active oxygen, amino acid derivative induced by stress. In addition, it plays a major role in cell control defense and repair in damaged cells. In addition, it is also expressed in normal cell function and plays an essential role as a molecular chaperon involved in folding and meeting of proteins. Recently, the protein has been referred to as a stress protein (22).

HSP47 is present in endoplasmic reticulum of collagen producing cells; its functions are closely involved in maturation of collagen and intracellular transport. At present, it has been recognized as a collagen specific molecular chaperone. Furthermore, it 
has been used as a histochemical marker of collagen-producing cells in unstressed cells and healthy cells.

Periodontal ligament cells in the control group expressed HSP47 even though they were in the state of equilibrium. Moreover, only few cells in junctional epithelium and subepithelial connective tissue showed positive reaction. This means that HSP47 was co-expressed by cells in non-stress condition in which the protein was regulated at the transcription level (23). Moreover, Muraoka et al. (7), mentioned that the weak expression of HSP70 in normal tissues was considered to be involved in the maintenance of homeostasis in the periodontal tissue. This made us thought that the expression of HSP47 is constantly involved in physiological remodeling of periodontal ligament due to excessive occlusal load.

From day 1 in experiment 1, fibroblasts near the epithelial attachment expressed HSP47. From this, we thought that the expression was brought about the traction force on periodontal ligament due to excessive occlusal load. The findings were similar to the study of Muraoka et al. (7) where HSP70 was initially detected on tension side after applying orthodontic force. In this regard, the result suggests that HSP47 is initially involved in remodeling of collagen fibers on tension side upon mechanical stress application. At day 4, HSP47 increased especially in cells on alveolar bone. Compression of the periodontal ligament was evident since collagen fibers underwent repair. HSP47 began to appear prominently in endothelial cells at day 7. This suggests that congestion of capillaries was due to excessive occlusal load and maintenance of homeostasis was made possible by HSP47. Although there was no increase in congestion at day 4, HSP47 was still considered to be involved in the process. With continuous load, HSP47 continued to increase with its peak at day 14 . It can be inferred that collagen fibers continue to repair in the presence of traumatic occlusion. With continuous accumulation of collagen fibers, fibrosis may follow with enduring occlusal force.

At day 3 in experiment 2, HSP47 was slightly stronger compared to control group suggesting the progressive repair of damaged collagen. Moreover, the cells lining the alveolar bone continued to express HSP47 at day 4 even though the load was removed. HSP47 continued to increase at day 6 and then decreased at day 10. Thus, HSP47 tend to increase over time. However, at day 30, the expression was similar to the control group suggesting that the cells revert back to their equilibrium state. Previous studies have shown the increase in HSP expression due to mechanical stress and decrease upon mechanical load release. Likewise, Keagle et at. (22) assumed that
HSP47 was expressed by damaged epidermis during wound healing. Thus, damaged cells due to mechanical trauma also express HSP47. The continuous expression of HSP47 prevented the disturbance of epidermal cells, abnormal cell division, rupture of blood vessels and other occurrences such as apoptosis. The continuous HSP expression from day 1 to day 14 was believed to be a protective response. Abnormal function of HSP47 is seen in damaged collagen fibers in periodontitis.

In summary, persistent occlusal overload can destroy collagen fibers in the periodontal ligament. This was evident by the increased in HSP47 expression with the placement of micro plus screw. HSP47 is maintained in fibroblasts for repair of damaged collagen fibers. On the other hand, osteoclasts continue to increase although the load was released. The osteoclasts that appeared on the alveolar bone surface were likely due to sustained activity. The increase in osteoclasts was estimated to occur after load application at day 4. HSP47 continued to increase until day 6 in experiment 2 but then reduced at day 10. Therefore, HSP47 appears after a period of certain activities to repair damaged collagen fibers, and the activity was returned to a state of equilibrium at day 30 with significantly diminished expression.

\section{Acknowledgment}

This study was supported by Grants-in-Aid for Scientific Research \# 26463104, \# 25463204 and \# 26861804 from the Japan Society for the Promotion of Science.

\section{Competing Interests}

The authors have declared that no competing interest exists.

\section{References}

1. Takaya T, Mimura H, Matsuda S, Nakano K, Tsujigiwa H, Tomita M, Okafuji $\mathrm{N}$, Fujii T, Kawakami T. Cytological kinetics of periodontal Ligament in an experimental occlusal trauma model. Int J Med Sci. 2015; 12: 544-51.

2. Pan H, Halper J. Regulation of heat shock protein 47 and type I procollagen expression in avian tendon cells. Cell Tissue Res. 2003; 311: 373-82.

3. Merryman WD, Youn I, Lukoff HD, Krueger PM, Guilak F, Hopkins RA, Sacks MS. Correlation between heart valve interstitial cell stiffness and transvalvular pressure: implications for collagen biosynthesis. Am J Physiol Heart Circ Physiol. 2006; 290: H224-31.

4. Oguro A1, Sakurai T, Okuno M, Nagata K, Atomi Y. The change of HSP47, collagen specific molecular chaperone, expression in rat skeletal muscle may regulate collagen production with gravitational conditions. Biol Sci Space. 2004; 18: 150-1.

5. Muraoka R, Nakano K, Kurihara S, Yamada K, Kawakami T. Immunohistochemical expression of heat shock proteins in the mouse periodontal tissues due to orthodontic mechanical stress. Eur J Med Res. 2010; 15: 475-82.

6. Muraoka R, Nakano K, Matuda H, Tomita M, Okafuji N, Kurihara S, Yamada $\mathrm{K}$, Kawakami T. Immunohistochemical observation of heat shock proteins expression in mouse periodontal tissues due to orthodontic mechanical stress. J Hard Tissue Biol. 2009; 18: 193-7.

7. Muraoka R, Nakano K, Matuda H, Tomita M, Okafuji N, Yamada K, Kawakami T. A consideration on the rode of HSP70 appearing in the periodontal tissues due to experimental orthodontic force. J Hard Tissue Biol. 2011; 20: 275-82. 
8. Fujii T, Takaya T, Mimura H, Osuga N, Matuda S, Nakano K. Experimental model of occlusal trauma in mouse periodontal tissues. J Hard Tissue Biol. 2014; 23: 377-80.

9. Svanberg G. Influence of trauma from occlusion on the periodontium of dog with normal or inflamed gingivae. Odontol Revy. 1976; 25: 165-78.

10. Stahl SS. Accommodation of the periodontium to occlusal trauma and inflammatory periodontal disease. Dent Clin Noth Am. 1975; 19: 531-42.

11. Lindhe J, Ericsson I. The influence of trauma from occlusion o reduced but healthy periodontal tissues in dogs. J Clin Periodontol. 1976; 3: 110-22.

12. Biancu S, Ericsson I, Lindhe J. Periodontal ligament tissue reactions to trauma and gingival inflammation. An experimental study in the beagle dog. J Clin Periodontol. 1995; 22: 772-9.

13. Glickman I, Smulow JB. Effect of excessive occlusal forces upon the pathway of gingival inflammation in humans. J Periodontol. 1965; 36: 141-7.

14. Waerhaug J. The Infrabony poket and its relationship to trauma from occlusion and subgingival plaque. J Periodontol. 1979; 7: 355-65.

15. Lindhe J, Svanberg G. Influence of trauma from occlusion on progression ofexperimental periodontitis in the beagle dog. J Clin Periodontol. 1974; 1: 3-14.

16. Ericsson I, Lindhe J. The effect of elimination of jiggling forces on periodontally exposed teeth in the dog. J Periodontol. 1982; 53: 562-7.

17. Svanberg G, Lindhe J. Vascular reactions in the periodontal ligament incident to trauma from occlusion. J Clin Periodontol. 1974; 1: 58-69.

18. Budtz-Jorgensen E. Occlusal dysfunction and stress. An experimental study in macaque monkeys. J Oral Rehabil. 1981; 8: 1-9.

19. Zhang G, Huang $X$, Herring SW. Effect of unilateral bite splint on mastication in the miniature pig. J Oral Rehabil. 1994; 21: 613-22.

20. Sodeyama T, Maeda T, Takano Y, Hara M. Responses of periodontal nerve terminals to experimentally induced occlusal trauma in rat molars; An immunohistochemical study using PGP 9.5 antibody. J Periodontal Res. 1996; 31: 235-48.

21. Kaku M, Uoshima K, Yamashita Y, Miura H. Investigation of periodontal ligament reaction upon excessive occlusal load-osteopontin induction among periodontalligament. J Periodont Res. 2005; 40: 59-66.

22. Keagle, JN, Welch WJ, Young DM. Expression of heat shock proteins in a linear rodent wound. Wound Rep Reg. 2001; 9: 378-85.

23. Koide T and Nagata K. HSP47, Molecular chaperon which Interacts with procollagens in the endoplasmic reticulum. Connect Tissue. 1998; 30: 307-11. 